\title{
Integrated Detectors for Embedded Optical Interconnections on Electrical Boards, Modules, and Integrated Circuits
}

\author{
Sang-Yeon Cho, Student Member, IEEE, Sang-Woo Seo, Student Member, IEEE, Martin A. Brooke, Member, IEEE, \\ and Nan M. Jokerst, Fellow, IEEE
}

\begin{abstract}
Significant opportunities exist for optical interconnections at the board, module, and chip level if compact, low-loss, high-data-rate optical interconnections can be integrated into these electrical interconnection systems. To create such an integrated optoelectronic/electronic microsystem, mask-based alignment of the optical interconnection waveguide, optoelectronic active devices, and interface circuits is attractive from a packaging alignment standpoint. This paper describes an integration process for creating optical interconnections which can be integrated in a postprocessing format onto standard boards, modules, and integrated circuits. These optical interconnections utilize active thin-film optoelectronic components embedded in the waveguide/interconnection substrate, thus eliminating the need for optical beam turning elements and their alignment, and providing an electrical output on the substrate from an optical interconnection. These embedded optical interconnections are reported herein using BCB polymer optical waveguides with embedded InGaAs-based thin-film inverted metal-semiconductor-metal (I-MSM) photodetectors on an $\mathrm{Si}$ substrate. These interconnections have been fabricated and tested, and the coupled optical signal from the waveguide to the embedded photodetector was theoretically modeled at $\mathbf{5 6 . 4 \%}$, which was supported by an experimental estimate of $\mathbf{4 7 . 8 \%}$. The measured full-width at half maximum of the electrical pulse from the MSM photodetector embedded in the waveguide was 16.73 ps for an input 500 -fs optical laser pulse.
\end{abstract}

Index Terms-Embedded photodetector, metal-semiconductor-metal, optical interconnection.

\section{INTRODUCTION AND MOTIVATION}

W ITH THE rapidly rising aggregate data rates and the decreasing size of electronic systems, conventional electrical interconnection faces numerous challenges at the board, module, and chip level. Power consumption, signal integrity (jitter, delay, skew), and area are factors which are in conflict in high performance electrical interconnection systems, and thus face tradeoffs. Resolution of these conflicts can be approached through innovative architectural and design approaches, or through physical technological innovation. Improvements in silicon integrated circuit technology and innovative designs, such as equalization [1] and advanced

Manuscript received September 03, 2002; revised October 01, 2002. This work was supported by the National Science Foundation Electronic Packaging Research Center and the State of Georgia Yamacraw Program.

The authors are with the National Science Foundation Packaging Research Center, School of Electrical and Computer Engineering, Georgia Institute of Technology, Atlanta, GA 30332-0250 USA.

Digital Object Identifier 10.1109/JSTQE.2002.806724 signaling techniques [2], have historically provided many of the gains in system performance to date [3]. However, underlying physical limitations to the interconnection technology still exist, and will ultimately force physical technology changes if performance gains are to continue.

The performance breakpoints and material systems differ for board- and chip-level interconnect. At the board level, the technology material options for electrical interconnect include epoxy, polymer, silicon, and ceramic. High-performance materials and structures for boards are more expensive and may suffer from miniaturization limitations. Thus, extending the performance capabilities of low-cost, widely implemented technologies such as polymers and epoxy (e.g., FR4) is an attractive alternative to higher cost board materials. If low-loss, wide-bandwidth, compact optical interconnections could be implemented at the board/module level with simple interface circuits, this may eliminate the need for large equalization circuits and large geometry transmission lines, thus enabling high-data-rate, small form factor interconnections on low-cost epoxy and polymer boards.

At the integrated circuit level, dimensional constraints prohibit some of the interconnect approaches utilized at the board level, such as transmission lines and equalization. Thus, material advances are critical, and hence the move in the silicon CMOS industry to copper lines and low dielectrics for interconnect. Even with these material advances, current microprocessor technology requires multiple repeaters and active feedback (e.g., phase locked loops) to stabilize clock skew and jitter to achieve high-speed signal propagation on global electrical clock lines. In a noisy digital environment, these interconnect signal integrity enhancing circuits actually add jitter to the signal [4], [5]. If low-loss, wide-bandwidth, compact optical interconnections could be implemented at the chip level with simple interfaces, this could improve the jitter performance of the interconnect by eliminating the need for repeaters and phase-locked loops.

\section{OPTICAL INTERCONNECTION INTEGRATION AND SYSTEM OPTIONS}

The integration of the optical signals into an electrical interconnection system is a topic of intensive study. A subset of optical interconnect approaches includes free space interconnects with diffractive optical elements [6], silicon optical bench interconnects [7], and guided wave interconnections, 


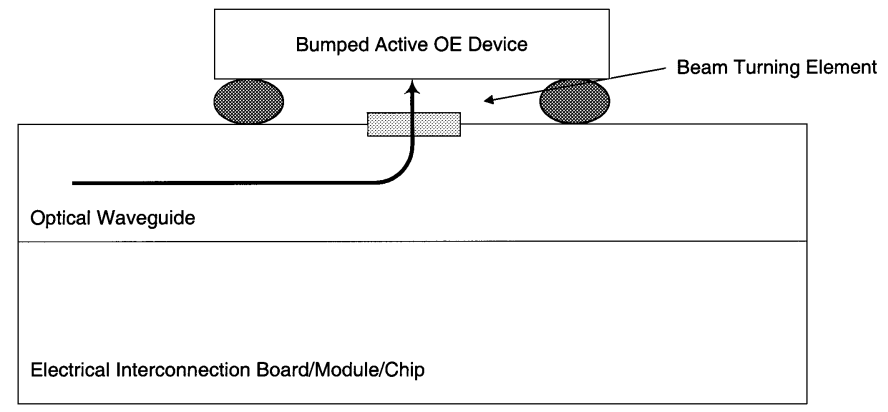

(a)

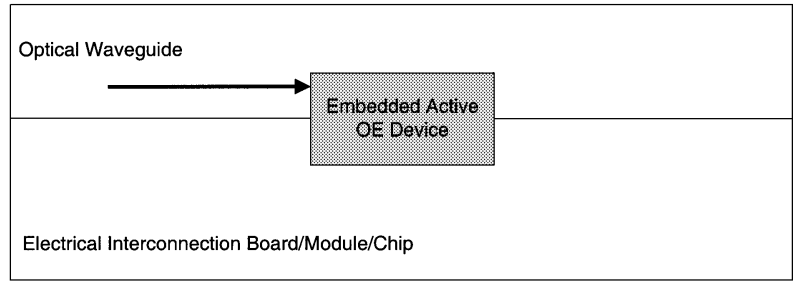

(b)

Fig. 1. Illustration of the optical signal coupling schemes in an integrated planar lightwave circuit in an electrical interconnection system. (a) Coupling with optical beam turning elements, such as diffractive optical elements, or micro-mirrors. (b) Evanescent field or direct coupling.

including substrate guided mode interconnects [8], fiber optic waveguides [9], and integrated waveguides [10]. Summary articles on this topic are available in the literature [11]. There are three fundamental technology areas which affect an optical interconnection: the interface electronics, the optoelectronic active devices (including emitters, detectors, modulators), and the optical passives (including the interconnection media and beam manipulation devices). This paper will focus on the integration of active optoelectronic components with waveguide optical interconnections and illustrate how electronic interface circuits can be integrated with these optical interconnections.

Electrical boards, modules, and integrated circuits are essentially planar, and thus, a planar waveguide is attractive for integration from a topography standpoint, with the system designer "turning" the optical signal or the electrical signal, as illustrated in Fig. 1. Optical beams can be routed in $90^{\circ}$ turns using mirrors or gratings and can be turned into either flip-chip optical/optoelectronic devices [Fig. 2(a)] or onto flip-chip optoelectronic integrated circuits (OEICs) [12] [Fig. 2(b)]. One drawback to this approach is that the alignment constraints in the optical system become tighter with increasing speed. Alternatively, the optical signals can originate and/or terminate in the waveguide without optical beam turning perpendicular to the plane of the substrate. Fig. 3 illustrates options for embedding a detector in a waveguide, including embedding in the core [Fig. 3(c) and (d)] and in the cladding [Fig. 3(e) and (f)]. Planar lightwave circuits (PLCs) with optical interconnections have been reported in compound semiconductors using waveguides and OE devices [13]-[15], demonstrating high coupling efficiency. Translating these ideas to polymer waveguides for optical interconnections compatible with board, module, and integrated circuit technology is of great interest.

There are two basic integration approaches to OE embedded waveguide interconnects using polymers. The first utilizes $\mathrm{OE}$ devices made from the same material as the electrical interconnection substrate. Using polymer waveguides integrated onto $\mathrm{Si}$ [16] or GaAs [17], [18] substrates which have photodetectors fabricated in the substrate, embedded waveguide interconnections have been demonstrated. The second integration approach utilizes thin-film OE devices (with the substrate removed) which can be bonded to any host substrate, including epoxy and polymer boards (such as FR4), and the photodetectors (PDs) are embedded directly into the waveguide, as illustrated in Fig. 3(c) and (d), or embedded in the cladding, as in Fig. 3(e) and (f).

Using this optoelectronic integration technology for $\mathrm{OE}$ devices embedded in waveguide interconnections, the designer has the option to create an optical interconnection on the board/module/chip which has exclusively electrical inputs and/or outputs (but can have optical inputs/outputs as well). One type of optical input which can be implemented to realize such a planar interconnect with a source in the waveguide is embedded thin-film edge-emitting lasers (at wavelengths including $850,980,1300$, and $1550 \mathrm{~nm}$ ) which emit directly into the waveguide structure. The use of vertical-cavity surface-emitting lasers (VCSELs) necessitates the use of beam-turning elements (note that the embedded thin-film $\mathrm{OE}$ device technology described in this paper can also be applied to GaAs-based devices such as PDs [19]). The planar lightwave circuit optical interconnections with embedded emitters and detectors may eliminate the need for optical beam-turning elements which route the beam perpendicular to the surface of the board/module/chip and reduces waveguide to active $\mathrm{OE}$ device optical alignment to an assembly step with sequentially aligned masking steps, which mimics integrated circuit fabrication. Connecting the interface circuits directly to the active $\mathrm{OE}$ devices can be realized through either wire bonding [Fig. 3(a)] or bump bonding [Fig. 3(b)] or through electrical substrate leads. The assembly tradeoff that is inherent in the OE device embedded waveguide interconnection is that the $\mathrm{OE}$ devices are bonded directly to the substrate rather than bumped to the substrate. One advantage of this approach is that the embedded OE device waveguide interconnections can be integrated onto the top of a fabricated electrical interconnection substrate through postprocessing, so optical interconnections can be implemented without impacting current board/module/chip fabrication lines. Finally, at the board/module level, the optical interconnects can be tested before chipset integration to enhance yield.

This paper describes the integration of independently fabricated waveguides, embedded thin film-film PDs, and a standard $\mathrm{Si}$ substrate. The integration processes described herein are also being applied to high-temperature epoxy FR4 boards. Presented herein are the integration processes and measurements for a thin-film ( $\sim 1-\mu$ m-thick) InGaAs PD embedded in a $\mathrm{BCB}$ polymer waveguide integrated onto an $\mathrm{Si}$ interconnection substrate, as shown in Fig. 3(d). The use of thin-film PDs (with the growth substrate removed) enables the PDs to be bonded and electrically connected to the $\mathrm{Si}$ interconnection substrate and embedded in the polymer optical waveguides, with the optical signal directly or evanescently coupled from the waveguide into the PD without the use of beam turning elements. Thus, the output signal from the substrate is an 


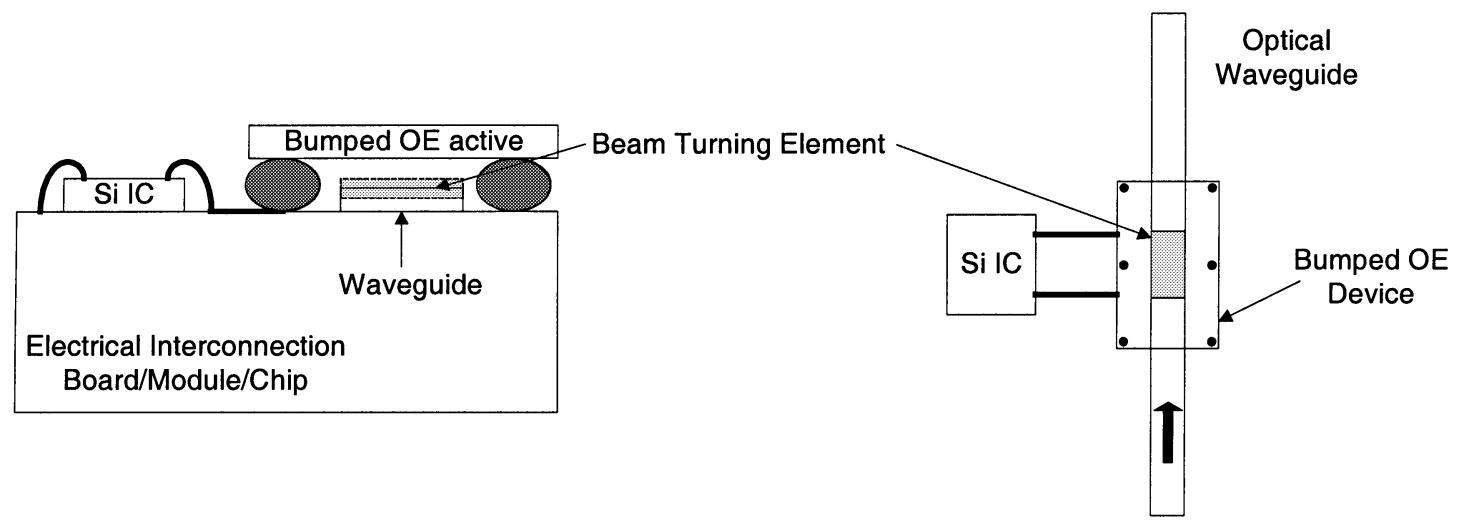

(a)

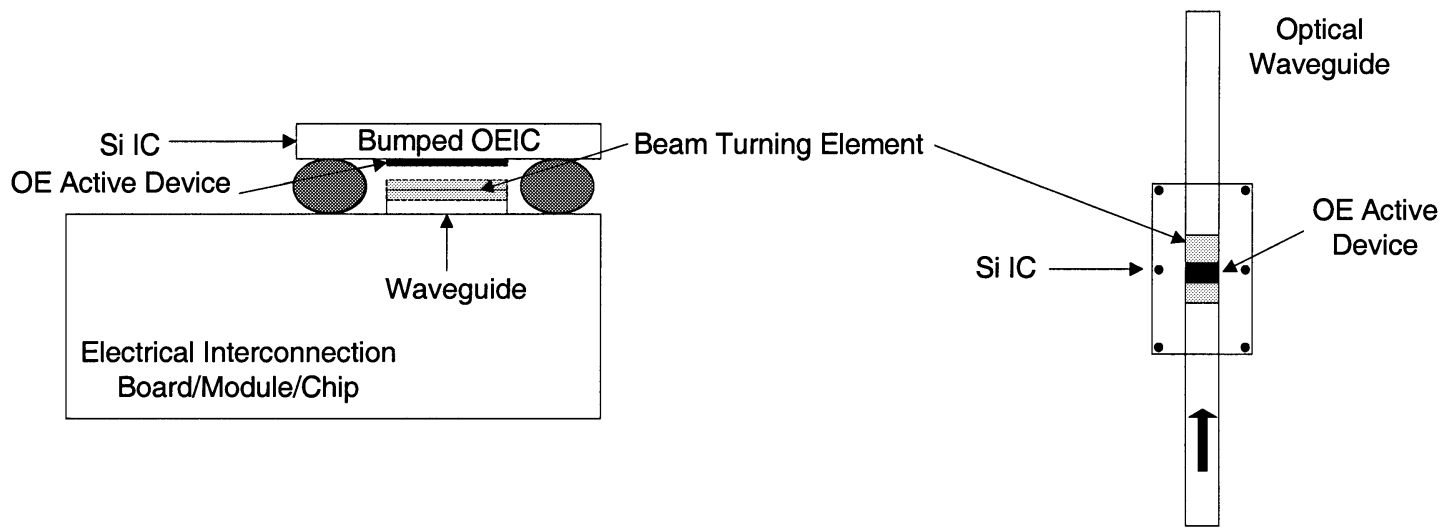

(b)

Fig. 2. Cross section and top view of optical and electrical signal routing options in integrated planar lightwave circuit interconnection in conventional electrical interconnection substrates. (a) Waveguide with beam-turning element produces surface normal optical output beam detected by bumped OE active device, with an $\mathrm{Si}$ interface integrated circuit wirebonded onto the electrical interconnection substrate. (b) Waveguide with beam-turning element produces surface normal optical output beam detected by bumped OEIC, with the active thin-film device integrated directly onto the Si interface integrated circuit. This OEIC is bump bonded to the electrical interconnection substrate.

electrical photodetector current, not a vertically outcoupled optical signal, and a receiver can be bonded directly to the PD [Fig. 3(a) and (b)]. Using this technology, thin-film OE devices, optical waveguides, and electrical interconnection media can be optimized separately for optimal signal distribution for board-, module-, and chip-level interconnections.

\section{PD AND WAVEGUIDE INTEGRATION}

To embed a thin-film InGaAs PD in a polymer waveguide, as illustrated in Fig. 3(d), the PD fabrication and waveguide fabrication are co-optimized. The structure demonstrated in this paper is a photodetector embedded in a $\mathrm{BCB}$ waveguide with $1 \mu \mathrm{m}$ of BCB core below the PD and $5.3 \mu \mathrm{m}$ of BCB core above it. To begin the integration, on the $\mathrm{Si}$ substrate (which is the electrical interconnection substrate), a $3-\mu \mathrm{m}$ layer of $\mathrm{SiO}_{2}$ was deposited onto the $\mathrm{Si}$ using plasma enhanced chemical vapor deposition (PECVD), followed by a spin-coated $1-\mu \mathrm{m}$-thick Benzocyclobutene (BCB) core layer followed by a cure at $240{ }^{\circ} \mathrm{C}$ for $1 \mathrm{~h}$. The 3- $\mu$ m-thick $\mathrm{SiO}_{2}$ layer acts as a cladding and buffer layer for the BCB waveguide. Contact pads of Ti/Au (400 $\AA$ / $5000 \AA$ ) were deposited and patterned on this substrate. Next, the thin-film PDs were separately fabricated and bonded to the pads on the Si substrate.
The thin film PDs were metal-semiconductor-metal (MSM) photodetectors which had the substrate removed, and the fingers on the bottom of the device. These are called inverted MSMs (I-MSMs), which have a higher surface normal responsivity than conventional MSMs since the finger shadowing is eliminated [20], and, when the I-MSMs and optical mode intensity distribution in the waveguide is co-optimized, can offer high-speed, high-responsivity operation with a large area coupled with low capacitance. MSMs have larger area per unit capacitance than PiN devices at the same speed (for speeds over roughly $500 \mathrm{MHz}$ ), which make them attractive candidates for high-speed operation in waveguide interconnection systems. Transimpedance amplifier interface circuits (TIAs) can only accept limited input capacitance from the PD, and, as speeds rise, $\mathrm{PD}$ areas decrease. Thus, to ensure adequate input signal to the TIA, large-area (i.e., more coupled signal), low-capacitance PDs are essential.

The I-MSM photodetectors were independently fabricated and subsequently bonded to the metal contact pads on the $\mathrm{BCB} / \mathrm{SiO}_{2} / \mathrm{Si}$ substrate. The as-grown MSM material was InP/InGaAs stop etch layer (2000 $\AA) / \mathrm{Al}_{0.48} \mathrm{In}_{0.52}$ As cap layer $(400 \AA) / \mathrm{Al}_{0.48} \mathrm{In}_{0.52}$ As graded to $\operatorname{In}_{0.53} \mathrm{Ga}_{0.47} \mathrm{As}(600 \AA) /$ $\mathrm{In}_{0.53} \mathrm{Ga}_{0.47} \mathrm{As}(0.74 \mu \mathrm{m}$ thick absorbing layer $) / \mathrm{In}_{0.53} \mathrm{Ga}_{0.47} \mathrm{As}$ graded to $\mathrm{Al}_{0.48} \mathrm{In}_{0.52} \mathrm{As}(600 \AA) / \mathrm{Al}_{0.48} \mathrm{In}_{0.52} \mathrm{As}$ cap layer 


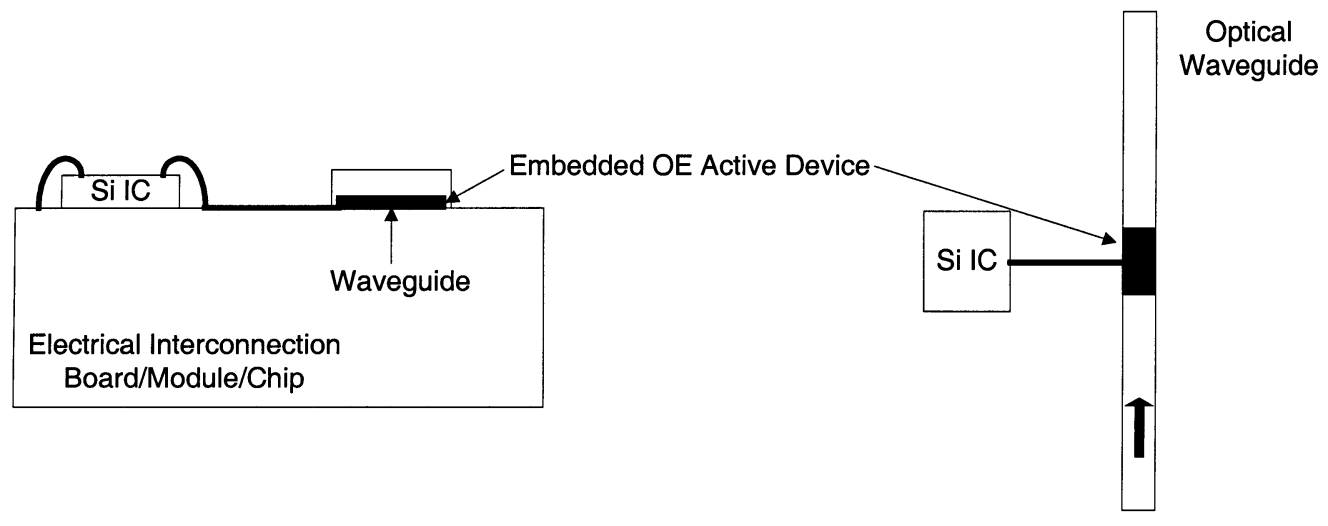

(a)

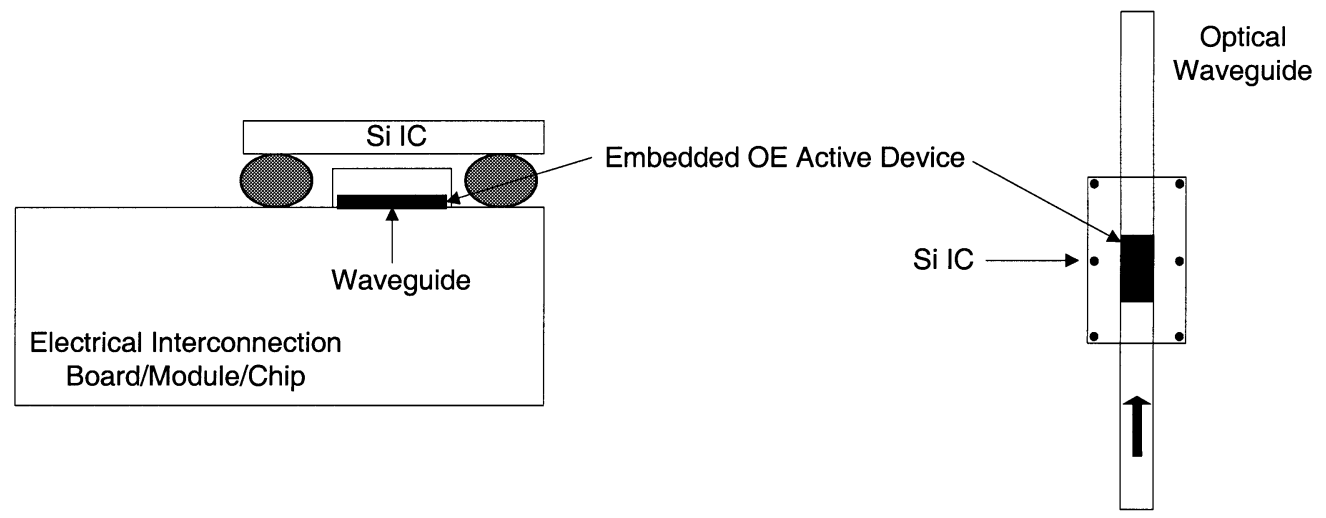

(b)

\begin{tabular}{|l|r|}
\hline Optical Waveguide & Core \\
\hline & \\
\hline $\begin{array}{l}\text { Electrical Interconnection } \\
\text { Board/Module/Chip }\end{array}$ & \\
\hline
\end{tabular}

(c)

\begin{tabular}{|lc|}
\hline Optical Waveguide & Core \\
\hline & \\
\hline $\begin{array}{l}\text { Electrical Interconnection } \\
\text { Board/Module/Chip }\end{array}$ & \\
\hline
\end{tabular}

(e)

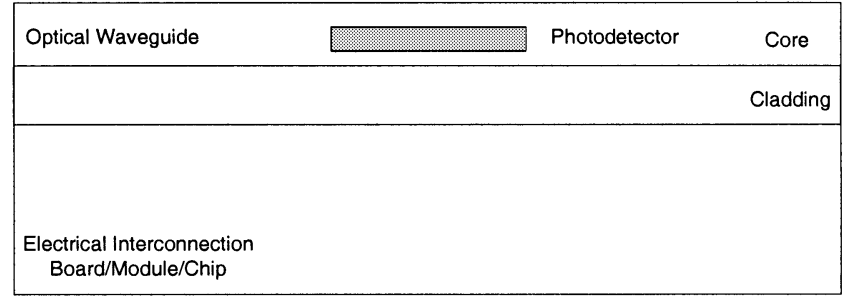

(d)

\begin{tabular}{|c|c|c|}
\hline Optical Waveguide & & Core \\
\hline & $\because$ Photodetector & Cladding \\
\hline $\begin{array}{l}\text { Electrical Interconnection } \\
\text { Board/Module/Chip }\end{array}$ & & \\
\hline
\end{tabular}

(f)

Fig. 3. Cross section and top view of optical and electrical signal routing options in integrated planar lightwave circuit interconnection with embedded active $\mathrm{OE}$ devices in conventional electrical interconnection substrates. (a) Waveguide with embedded photodetector which detects input optical beam with photocurrent output to the Si interface integrated circuit wirebonded onto the electrical interconnection substrate. (b) Waveguide with embedded photodetector which detects input optical beam with photocurrent output to the $\mathrm{Si}$ interface integrated circuit which is bump bonded to the electrical interconnection substrate. Embedded active OE device integration options shown for photodetectors, which include (c), (d) direct coupling and (e), (f) evanescent field coupling.

(400 $\AA$ ). Schottky contacts of $40-\AA \mathrm{Pt} / 350-\AA \mathrm{Ti} / 400-\AA$ $\mathrm{Pt} / 2500-\AA \mathrm{Au}$ were deposited, and interdigitated fingers $100 \mu \mathrm{m}$ long with $2-\mu \mathrm{m}$ finger width and $2-\mu \mathrm{m}$ finger spacing were patterned on a $100 \mu \mathrm{m} \times 150 \mu \mathrm{m}$ detection area. The 400- $\AA$-thick Pt layer is a diffusion barrier for the Au into the Ti during the polymer waveguide thermal curing process [21]. By utilizing the Pt diffusion barrier on the MSM, the Schottky barriers are not degraded by the waveguide thermal process and, thus, the dark current is not degraded by the integration process. Next, each MSM device was mesa etched down to the InP substrate layer using citric: $\mathrm{H}_{2} \mathrm{O}_{2}$ (10:1). The MSM mesas were protected with Apiezon $\mathrm{W}$ wax and immersed in $\mathrm{HCl}$ to selectively remove the InP substrate, stopping on the InGaAs layer [22]. The thin-film MSM mesa devices in the Apiezon 
W wax were next bonded to a mylar transfer diaphram. The Apiezon $\mathrm{W}$ was dissolved with trichloroethylene, leaving the thin-film MSM devices bonded to the mylar transfer diaphram. The final thickness of the MSM devices was $0.9 \mu \mathrm{m}$.

Next, the thin-film MSMs were transferred onto the $\mathrm{BCB} / \mathrm{SiO}_{2} / \mathrm{Si}$ substrate, and the waveguide integration to embed the I-MSMs was completed. The MSMs were transferred using the mylar transfer diaphragm [23], and were bonded to the metal pads on the $\mathrm{BCB} / \mathrm{SiO}_{2} / \mathrm{Si}$ substrate, thus inverting the device (creating the I-MSM) [24], [25] . This metal (I-MSM pad) to metal $\left(\mathrm{BCB} / \mathrm{SiO}_{2} / \mathrm{Si}\right.$ substrate $\left.\mathrm{pad}\right)$ bond is an electrically conducting, mechanically stable bond formed using a low temperature thermal anneal of 10 minutes at $150{ }^{\circ} \mathrm{C}$, which was performed after the device was bonded to the contact pads. The waveguide fabrication process was then continued to embed the photodetector in the polymer waveguide. BCB was used previous as the first layer of the waveguide core layer, and was next, again, spin coated onto the I-MSM/BCB $/ \mathrm{SiO}_{2} / \mathrm{Si}$ sample and cured at $240{ }^{\circ} \mathrm{C}$ for $1 \mathrm{~h}$, with a resulting total $\mathrm{BCB}$ core waveguide thickness of $\sim 7 \mu \mathrm{m}$. Both BCB thermal cures were carried out in a nitrogen ambient to avoid oxidation of the $\mathrm{BCB}$, since oxidization can slightly increase the refractive index of the film [26]. Thus, the embedded I-MSM integrated has $1 \mu \mathrm{m}$ of BCB core below it and $6 \mu \mathrm{m}$ of BCB above it.

To complete the waveguide integration process, the BCB was polished and defined into channels. To minimize the scattering loss due to the surface roughness of the core layer, the BCB top layer was chemi-mechanically polished (CMP) using Rodel $3116 \mathrm{~B}$ and deionized water. The abrasive used for the slurry was $0.05 \mu \mathrm{m} \mathrm{Al}{ }_{2} \mathrm{O}_{3}$. The final thickness and surface roughness of the core layer were $6.3 \mu \mathrm{m}$ and $600 \AA$ respectively, as measured by a profilometer. The BCB planar waveguide was then patterned into a $100-\mu \mathrm{m}$-wide channel waveguide using a thick photoresist mask and $\mathrm{SF}_{6} / \mathrm{O}_{2}$ reactive ion etching. The width of the fabricated waveguide was designed to match the detection area $(100 \mu \mathrm{m} \times 150 \mu \mathrm{m})$ of the embedded I-MSM PD to maximize the coupling efficiency from the waveguide to the embedded PD. By defining the 100- $\mu \mathrm{m}$-wide BCB channel over the center of inverted MSM photodetector, the guided optical signal is incident on the high-electrical-field interdigitated finger region of the embedded I-MSM photodetector. Thus, photogenerated carriers caused by direct coupling from the waveguide into the PD are effectively swept into the interdigitated finger contacts. Fig. 4 is a photomicrograph of the fabricated I-MSM PD embedded in the $\mathrm{BCB}$ polymer waveguide on the $\mathrm{SiO}_{2} / \mathrm{Si}$ substrate.

\section{MEASUREMENTS}

The I-MSM dark current and photoresponse were measured before and after waveguide fabrication. The photoresponse before integration was measured in a surface normal configuration and, after integration, in the embedded waveguide configuration. The dark current and photoresponse of the I-MSM were measured using a Keithley 236 source measurement unit to measure the current output of the I-MSM. A fiber pigtailed laser diode connected to a $62.5-\mu \mathrm{m}$ multimode fiber was used

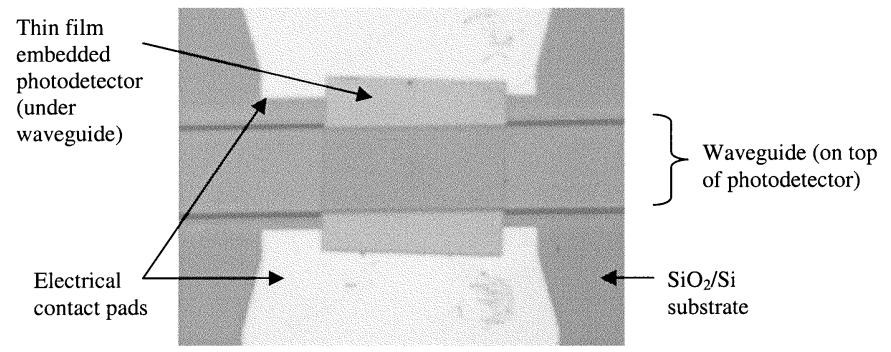

(a)

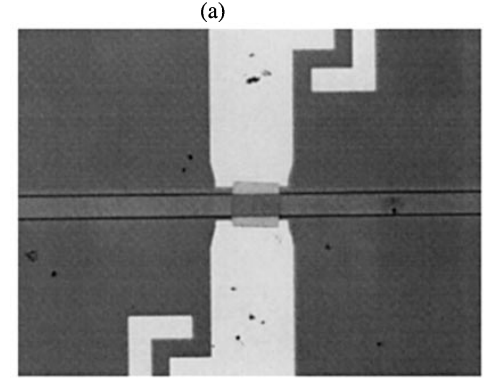

(b)

Fig. 4. Photomicrographs of a thin film I-MSM photodetector embedded in a $\mathrm{BCB}$ polymer waveguide on $\mathrm{SiO}_{2} / \mathrm{Si}$ electrical interconnection substrate: (a) close-up and (b) farther from the structure.

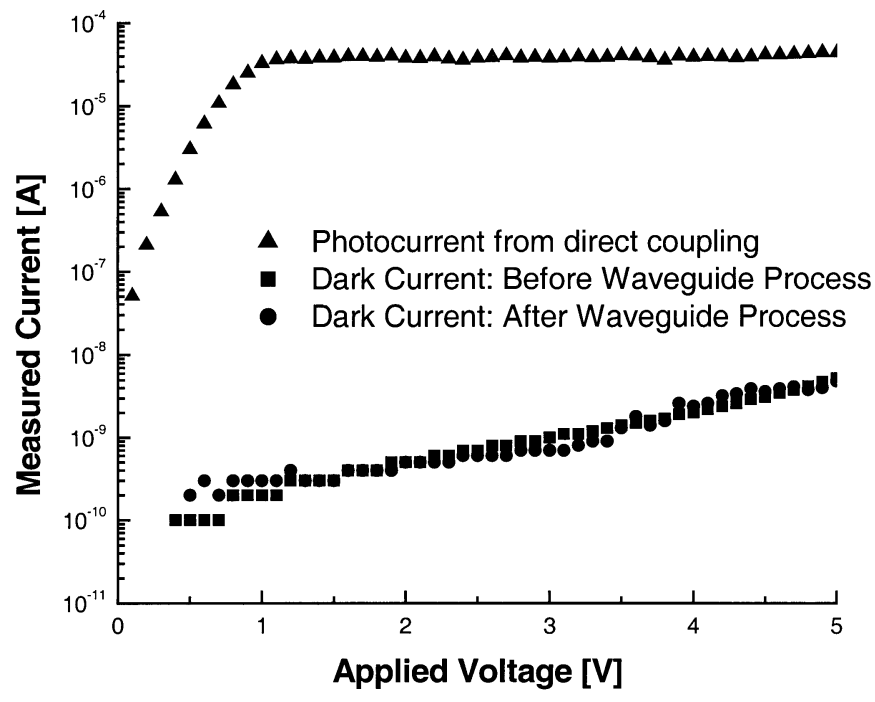

Fig. 5. Dark current and photocurrent measurements of the thin-film I-MSM photodetector embedded in a $\mathrm{BCB}$ polymer waveguide on an $\mathrm{SiO}_{2} / \mathrm{Si}$ electrical interconnection substrate shown in Fig. 4 . $\mathbf{\Delta}$ : measured photocurrent using direct waveguide coupling; $\mathbf{\square}$ : measured dark current before the waveguide process; $\bullet$ : measured dark current after the BCB waveguide integration process.

for the optical input for the surface normal responsivity. The optical power output from the fiber was $1.54 \mathrm{~mW}$, measured with a calibrated Newport 1835 optical power meter. The I-MSM surface normal responsivity was measured before the waveguide was integrated onto the detector, and, without an AR coating, had a measured responsivity of $0.38 \mathrm{~A} / \mathrm{W}$ at $5 \mathrm{~V}$. After the waveguide integration, to test the waveguide coupling, the Si substrate was cleaved to produce an endface on the polymer waveguide, and a single mode optical fiber ( core diameter $=9 \mu \mathrm{m}$, Numerical Aperture $(N A)=0.13$ ) was endface coupled to the polymer waveguide. Fig. 5 shows 
the measured dark current before and after the polymer optical waveguide process, as well as the photocurrent due to the coupling from the waveguide to the I-MSM PD embedded in the waveguide. The dark current, which is $5.2 \mathrm{nA}$ at $5 \mathrm{~V}$ before waveguide integration and $4.8 \mathrm{nA}$ at $5 \mathrm{~V}$ after waveguide integration, has improved slightly after the waveguide process, which may be due to low-temperature annealing of the I-MSM Schottky contacts. The photocurrent at $5 \mathrm{~V}$ was $42.02 \mu \mathrm{A}$. Thus, the optical signal in the waveguide has been successfully coupled into the embedded photodetector. Later in this paper, the coupling efficiency from the waveguide into the embedded PD will be discussed.

The propagation loss of the integrated channel polymer waveguide was measured using the optical fiber scanning method. The probing large-core multimode fiber (core diameter $=600 \mu \mathrm{m}, N A=0.37$ ) measures the scattered optical signal from the fabricated channel waveguide. The probing optical fiber was set at the same height (within a $\mathrm{cm}$ ) from the sample while 300 scanning points (using a Coherent motorized micro-positioner), which were measured perpendicular to the waveguide in $10-\mu \mathrm{m}$ increments. These 300 points constitute one scan line. There were 300 of these scan lines measured, each separated by $10 \mu \mathrm{m}$. The propagation loss per unit distance was calculated by the slope of the linear least square regression line of the average collected (scattered) optical power from the waveguide before the detector, as measured through the probing fiber. Using this method, the estimated propagation loss of the multimode BCB polymer channel waveguide was $0.36 \mathrm{~dB} / \mathrm{cm}$ at a wavelength of $1.3 \mu \mathrm{m}$. This estimate includes intrinsic material, structural, and other possible propagation loss sources of the polymer optical waveguide, which is consistent with other reported results for BCB waveguides [27], [28]. This measurement assumes uniform scattering along the fabricated optical channel waveguide.

The electrical pulse response to a short optical pulse was measured for the embedded thin film I-MSM photodetector. A short pulse fiber laser $(\lambda=1.55 \mu \mathrm{m})$ was used as the light source in this speed measurement. A 500 femtosecond full-width at halfmaximum (FWHM) optical pulse was endface coupled through a lensed single-mode optical fiber (minimum spot size $=5 \mu \mathrm{m}$ ) into the fabricated BCB polymer waveguide. The electrical response from the embedded thin film MSM photodetector was probed through a 40-GHz Cascade GS (Ground Signal) microwave probe. Then the electrical pulse was measured with a Tektronics $50 \mathrm{GHz}$ sampling oscilloscope. The electrical pulse response from the embedded I-MSM photodetector is shown in Fig. 6. The measured FWHM output of the I-MSM output pulse was 16.73 ps at $3.5 \mathrm{~V}$.

The measured pulse response of the embedded I-MSM photodetector is very fast, given the large area of this photodetector $(100 \mu \mathrm{m} \times 150 \mu \mathrm{m})$, in comparison to published MSM photodetectors which run at similar speeds, which are on the order of $30 \mu \mathrm{m}$ in diameter [29]-[31]. A variety of factors may explain this improvement in speed for the embedded I-MSM. MSMs which are attached to the growth substrate have electric field lines which radiate into the substrate, and thus, are not as well confined to the MSM absorbing region as the thin film embedded I-MSM, which is surrounded by

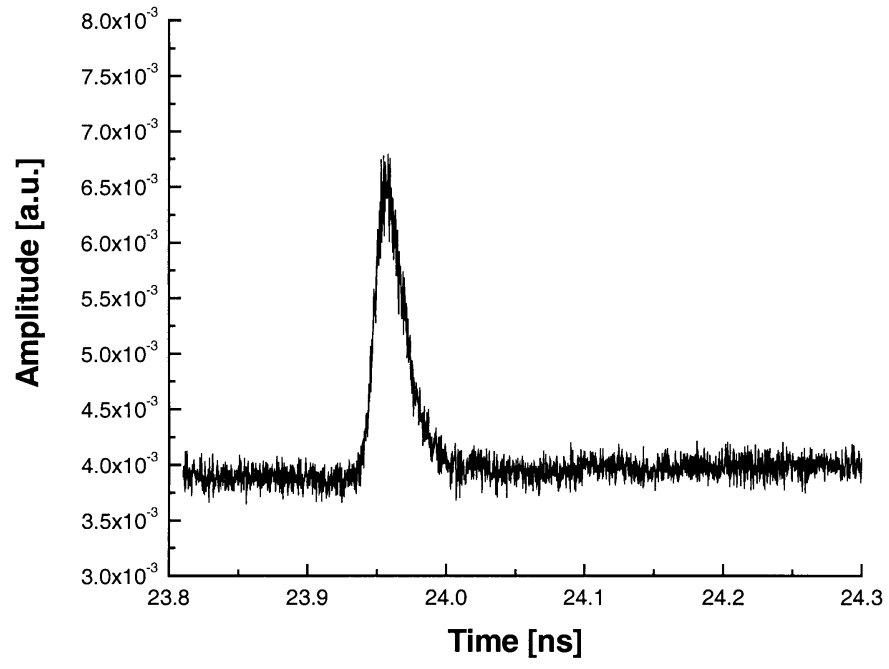

Fig. 6. The response of the embedded I-MSM photodetector in the BCB polymer waveguide to a 500-fs optical pulse.

the low-dielectric-constant $\mathrm{BCB}$ polymer material. Thus, the electric field confinement in the thin-film embedded I-MSM is better than in conventional MSMs on the growth substrate. Further theoretical analysis and measurements related to these issues will be discussed in detail in another publication. For high-speed optical interconnect, where detector size may limit the coupling efficiency, larger high-speed embedded PDs will produce the opportunity for higher coupling efficiencies.

\section{THEORETICAL ANALYSIS}

The coupling from the waveguide to the PD can be modeled and estimated, but it is difficult to precisely measure the coupling for the single embedded PD due to the difficulty in estimating the fiber to waveguide endface coupling efficiency. Herein, the waveguide to I-MSM coupling efficiency is theoretically calculated and estimated from measurement results. The optical signal coupling efficiency was theoretically calculated for the embedded I-MSM PD in the multimode waveguide structure based on the 2-D finite-difference beam propagation method (BPM). The high index contrast between the waveguide and the embedded I-MSM PD and the reflection at the embedded I-MSM PD interface was addressed using wide-angle and bidirectional BPM [32], [33]. These theoretical models were generated using RSoft, a BPM modeling software package. The estimated coupling efficiency from the $6.3-\mu \mathrm{m}$-thick multimode waveguide into the $150-\mu \mathrm{m}$-long embedded I-MSM PD was $56.4 \%$ using scalar wave analysis. This structure is not optimized; a more optimized structure, with only a decrease in waveguide thickness, would result in a coupling efficiency of over $80 \%$. Other more optimized waveguide/detector structures can yield over 95\% coupling efficiency and will be discussed in a later publication.

The coupling efficiency from the waveguide to the embedded I-MSM PD was also estimated through measurement. The measured output from the single-mode optical fiber was $1.54 \mathrm{~mW}$. Using a 3-D finite difference (FD) BPM, an estimated $16.7 \%$ of this optical signal was coupled into the waveguide if the fiber to waveguide endface separation is $10 \mu \mathrm{m}$, which 
is the estimated separation, and the waveguide shrinkage due to curing is $10 \%$. The coupling efficiency drops to $16.6 \%$ for a $20-\mu \mathrm{m}$ separation, so the coupling is not highly sensitive to the estimated fiber to endface separation within the limits of reasonable separation error, as measured by a high-magnification microscope. This estimate included the following inputs: waveguide $\mathrm{BCB}$ index of refraction $=1.53$ at $\lambda=1.3 \mu \mathrm{m}$ (based upon the $\mathrm{BCB}$ vendor data for the deposition conditions used), $\mathrm{SiO}_{2}$ index of refraction $=1.45$ at $\lambda=1.3 \mu \mathrm{m}$ (based upon the PECVD data for the deposition conditions used), fiber core refractive index $=1.5$, diameter $=9 \mu \mathrm{m}$. The fiber optical excitation mode is assumed to be a fundamental fiber mode which matches the fiber core diameter. The output and input faces of the fiber and waveguide, respectively, were assumed to be planar. The 3D FD BPM grid size was $0.01 \lambda$, and the operating wavelength was $1.3 \mu \mathrm{m}$. The optical signal travels $1.19 \mathrm{~cm}$ in the waveguide from the excitation endface to the detector, which causes a loss of $0.43 \mathrm{~dB}$ (using the measured $-0.36 \mathrm{~dB} / \mathrm{cm}$ measured propagation loss). Thus, the estimated optical power incident on the embedded detector in the waveguide is $0.23 \mathrm{~mW}$. To calculate the PD absorbed optical power, divide the photocurrent $(42.02 \mu \mathrm{A})$ from the embedded photodetector (when it is excited through coupling in the waveguide) by the PD surface normal responsivity $(0.38 \mathrm{~A} / \mathrm{W})$. Thus, $0.11 \mathrm{~mW}$ of optical power was absorbed by the photodetector. So, the estimated coupling efficiency is the absorbed power divided by the incident power: $47.8 \%$, which is on the same order as the theoretical coupling efficiency, which is $56.4 \%$. Although this estimate of the measured coupling efficiency is very rough, it does indicate that sufficient coupling can be achieved to create a viable interconnect. One of the attractive aspects of this integration technology is that the coupling can be intentionally varied through structural design so that the majority of the optical signal can be detected, or a fraction of the signal can be detected, thus reserving signal for subsequent PDs which can be located further down the optical path on the same waveguide for spatial division multiplexing.

\section{CONCLUSION}

Significant opportunities exist for optical interconnections at the board-, module-, and chip-level if compact, low-loss, high-data-rate optical interconnections can be integrated into these electrical interconnection systems. There are numerous approaches which implement optical interconnections into electrical systems. For such an integrated optoelectronic/electronic microsystem, mask-based alignment of the optical interconnection waveguide, optoelectronic active devices, and interface circuits is attractive from a packaging alignment standpoint. This paper describes an integration process for creating optical interconnections which can be integrated in a postprocessing format onto standard boards (including polymer and epoxy), modules, and integrated circuits. These optical interconnections utilize active optoelectronic components embedded in the waveguide/interconnection substrate, thus providing an electrical output to the user from an optical interconnection. These embedded optical interconnections have been demonstrated and reported herein using BCB polymer optical waveguides with embedded InGaAs-based thin-film I-MSM photodetectors on an $\mathrm{Si}$ substrate. These interconnections have been fabricated and tested, demonstrating over $47 \%$ coupling efficiency. The measured FWHM of the electrical pulse from the embedded MSM photodetector was $16.73 \mathrm{ps}$ for a $500-f s$ optical input laser pulse. The integration process reported herein for these embedded optical interconnections is consistent with integration onto electrical interconnection microsystems. Thus, there is promise for the incorporation of optical interconnections with standard electrical signals at the board, module, and integrated circuit level.

\section{ACKNOWLEDGMENT}

The authors would like to thank the staff of the Georgia Tech Microelectronics Research Center cleanroom for their support.

\section{REFERENCES}

[1] W. J. Dally and J. Poulton, "Transmitter equalization for 4-Gbps signaling," IEEE Micro, pp. 48-56, 1997.

[2] C. Yoo, "High-speed DRAM interface," IEEE Potentials, vol. 20, pp. 33-34, 2002.

[3] W. Ryu, J. Lee, H. Kim, S. Ahn, N. Kim, B. Choi, D. Kam, and J. Kim, "RF interconnect for multi-Gbit/s board-level clock distribution," IEEE Trans. Adv. Packag., vol. 23, pp. 398-407, 2000.

[4] K. A. Jenkins and J. P. Eckhardt, "Measuring jitter and phase error in microprocessor phase-locked loops," IEEE Des. Test. Comput., vol. 17, pp. 86-93, 2000.

[5] M. Horowitz, C.-K. K. Yang, and S. Sidiropoulos, "High-speed electrical singaling: Overview and limitations," IEEE Micro, pp. 12-24, 1998.

[6] S. K. Tewksbury and L. A. Hornak, "Optical clock distribution in electronic systems,” J. VLSI Signal Process., vol. 16, pp. 225-246, 1997.

[7] M. Rassaian and M. W. Beranek, "Quantitative characterization of 96.5Sn3.5Ag and 80Au20Sn optical fiber solder bond joints on silicon micro-optical bench substrates," IEEE Trans. Adv. Packag., vol. 22, pp. 86-93, 1999.

[8] S. J. Walker and J. Jahns, "Optical clock distribution using integrated free-space optics," Opt. Commun., vol. 90, pp. 359-371, 1992.

[9] P. J. Delfyett, D. H. Hartman, and S. Z. Ahmad, "Optical clock distribution using a mode-locked semiconductor laser-diode system," J. Lightwave Technol., vol. 9, pp. 1646-1649, 1991.

[10] B. Bihari, J. Gan, L. Wu, Y. Liu, S. Tang, and R. T. Chen, "Optical clock distribution in supercomputers using polyimide-based waveguides," in Proc. Opto. Intercon. VI., San Jose, CA, Jan. 1999, pp. 123-133.

[11] D. A. B. Miller, "Rationale and challenges for optical interconnects to electronic chips," Proc. IEEE, vol. 88, pp. 728-749, 2000.

[12] N. M. Jokerst, M. A. Brooke, J. Laskar, D. S. Wills, A. S. Brown, M. Vrazel, S. Jung, Y. Joo, and J. J. Chang, "Microsystem optoelectronic integration for mixed multisignal systems," IEEE J. Select. Topics Quantum Electron., vol. 6, pp. 1231-1239, 2000.

[13] S. Kollakowski, A. Strittmatter, E. Dröge, E. H. Böttcher, and Bimberg, "65 GHz InGaAs/InAlGaAs/InP waveguide-integrated photodetectors for the 1.3-1.55 $\mu \mathrm{m}$ wavelength regime," Appl. Phys. Lett., vol. 74, pp. 612-614, 1999.

[14] E. H. Böttcher, H. Pfitzenmaier, E. Dröge, S. Kollakkowski, A. Stittmatter, D. Bimberg, and R. Steingrüber, "Distributed waveguide-integrated InGaAs MSM photodetectors for high-efficiency and ultra-wideband operation," in Proc. IEEE 11th Int. Conf. Indium Phosphide and Related Materials, 1999, pp. 79-82.

[15] S. Kollakowski, E. Dröge, E. H. Böttcher, A. Strittmatter, O. Reimann, and D. Bimberg, "Waveguide-Integrated InP/InGaAs/InAlGaAs MSM photodetector for operation at 1.3 and $1.55 \mu \mathrm{m}$," in Proc. IEEE 10th Int. Conf. Indium Phosphide and Related Materials, 1998, pp. 266-268.

[16] C. H. Buchal, A. Roelofs, M. Siegert, and M. Löken, "Polymeric strip waveguides and their connection to very thin ultrafast metal-semiconductor-metal detectors," in Mat. Res. Soc. Symp. Proc., vol. 597, 2000, pp. 97-102.

[17] F. Gouin, L. Robitaille, C. L. Callender, J. Noad, and C. Almeida, "A $4 \times 4$ optoelectronic switch matrix integrating an MSM array with polyimide optical waveguides," Proc. SPIE, vol. 3290, pp. 287-295, 1997. 
[18] C. L. Callender, L. Robitaille, J. P. Noad, F. Gouin, and C. Almeida, "Optimization of metal-semiconductor-metal (MSM) photodetector arrays integrated with polyimide waveguides," Proc. SPIE, vol. 2918, pp. 211-221, 1997.

[19] O. Vendier, N. M. Jokerst, and R. Leavitt, "High efficiency inverted GaAs-Based MSM photodetectors," Electron. Lett., vol. 32, no. 4, pp. 394-395, Feb. 1996.

[20] O. Vendier, N. M. Jokerst, and R. P. Leavitt, "Thin-film inverted MSM photodetectors," IEEE Photon. Technol. Lett., vol. 8, pp. 266-268, 1996.

[21] D. G. Ivey, P. Jian, R. Bruce, and G. Knight, "Microstructural analysis of Au/Pt/Ti contacts to p-type InGaAs," J. Mat. Sci.: Mat. in Electron., vol. 6, pp. 219-227, 1995.

[22] N. M. Jokerst, M. A. Brooke, O. Vendier, S. Wilkinson, S. Fike, M. Lee, E. Twyford, J. Cross, B. Buchanan, and S. Wills, "Thin-film multimaterial optoelectronic integrated circuits," IEEE Trans. Comp., Packag., Manufact. Technol. B, vol. 1, pp. 97-106, 1996.

[23] N. M. Jokerst, "Hybrid integrated optoelectronics: Thin film devices bonded to host substrates," Int. J. High Speed Electron. Syst., vol. 8, pp. 325-356, 1997

[24] Y. Wang, S. Fike, M. G. Allen, and N. M. Jokerst, "Measurement of adhesion of GaAs thin films using the blister test," in Proc. Annu. Meeting Adhesion Soc., Orlando, FL, Feb. 1994.

[25] N. Jokerst, M. Brooke, O. Vendier, S. Wilkinson, S. Fike, M. Lee, E. Twyford, J. Cross, B. Buchanan, and S. Wills, "Thin film multimaterial optoelectronic integrated circuits," IEEE Trans. Comp., Packag., Manufact. Technol. B, vol. 19, no. 1, pp. 97-106, Feb. 1996.

[26] R. Kirchoff, C. Carriere, K. Bruza, N. Rondan, and R. Sammler, "Benzocyclobutenes: A new class of high performance polymer," J. Macro. Sci.-Chem., vol. 28, pp. 1079-1113, 1991.

[27] S. Wolff, A. R. Giehl, M. Renno, and H. Fouckhardt, "Metallic waveguide mirrors in polymer film waveguides," Appl. Phys. B, vol. 73, pp. 623-627, 2001

[28] G. Fischbeck, R. Moosburger, M. Topper, and K. Peterman, "Design concept for singlemode polymer waveguides," Electron. Lett., vol. 32, pp. 212-213, 1996

[29] Y. G. Zhang, A. Z. Li, and J. X. Chen, "Improved performance of InAlAs-InGaAs-InP MSM photodetectors with graded superlattice structure grown by gas source MBE," IEEE Photon. Technol. Lett., vol. 8, pp. 830-832, 1996

[30] S. Kollakowski, E. H. Bottcher, A. Strittmatter, and D. Bimberg, "Highspeed InGaAs/InAlGaGs/InP waveguide-integrated MSM photodetectors for 1.3-1.55 $\mu \mathrm{m}$ wavelength range," Electron. Lett., vol. 34, pp. $587-589,1998$

[31] E. H. Bottcher, E. Droge, A. Strittmatter, and D. Bimberg, "Polarizationinsensitive high-speed InGaAs metal-semiconductor-metal," Electron. Lett., vol. 33, pp. 912-914, 1997.

[32] R. Scarmozzino, A. Gopinath, R. Pregla, and S. Helfert, "Numerical techniques for modeling guided-wave photonic devices," IEEE J. Select. Topics Quantum Electron., vol. 6, pp. 150-162, 2000.

[33] G. R. Hadley, "Wide-angle beam propagation using Pade approximant operators," Opt. Lett., vol. 25, pp. 1426-1428, 1992.

Sang-Yeon Cho (S'02) received the B.S. degree and the M.S. degree in electrical and computer engineering from SungKyunKwan University, Korea, in 1996 and 1998, respectively, and the M.S. degree in electrical and computer engineering from the Georgia Institute of Technology (Georgia Tech), Atlanta, in 2000 . He is currently working toward the Ph.D. degree at Georgia Tech.

His research interest is in high-speed optical interconnection using thin-film active optoelectronic devices with polymer optical waveguides.
Sang-Woo Seo (S'02) received the B.S. degree in electrical and computer engineering from Ajou University, Korea, in 1997 and the M.S. degree from Kwangju Institute of Science and Technology (KJIST), Korea, in 1999. He is currently working toward the Ph.D. degree at the Georgia Institute of Technology, Atlanta.

He has experience in semiconductor laser devices at KJIST, and his current main interest is the development of thin-film detectors for multispectral imaging and high-speed optical interconnections.

Martin A. Brooke (S'85-M'86) received the B.E.(Elect.) degree (with first class honors) from Auckland University, New Zealand, in 1981 and the M.S. and Ph.D. degrees in electrical engineering from The University of Southern California, Los Angeles, in 1984 and 1988, respectively.

$\mathrm{He}$ is currently an Associate Professor of Electrical Engineering at the Georgia Institute of Technology, Atlanta. He has published more than 100 papers in trechnical journals and proceedings, and articles on his work have appeared in several trade publications. His expertise is in high-speed, high-performance signal processing. His current projects include learning neural network hardware development, neural network prediction of turbulent flow, focal plane image processing hardware development, 1-20-Gb/s digital CMOS transceiver circuits for low-cost fiber-optic communication, nonlinear filtering algorithms for telecommunications, nonlinear analog-to-digital converter design, accurate modeling of high-speed circuit parasitics, and statistically relevant device models for acurate prediction of high-performance integrated circuit yield. He holds 4 U.S. patents.

Prof. Brooke was awarded a National Science Foundation Research Inititiation Award in 1990 and the 1992 IEEE Midwest Symposium on Circuits and Systems Myril B. Reed Best Paper Award.

Nan M. Jokerst (S'83-M'88-SM'98-F'02) received the Ph.D. degree from the University of Southern California, Los Angeles, in 1989.

She is the Joseph M. Pettit Professor of Optoelectronics in the School of Electrical and Computer Engineering, Georgia Institute of Technology (Georgia Tech), Atlanta. She joined the Electrical Engineering faculty, Georgia Tech, in 1989. She is the Optoelectronics Thrust Leader for the Georgia Tech National Science Foundation Engineering Research Center in Electronic Packaging. She has published and presented over 175 papers, three book chapters, and has three patents awarded and two pending.

Dr. Jokerst is a Fellow of the Optical Society of America. She received an IEEE Millenium Medal in 2000 and has received the Harriet B. Rigas Education Award from the IEEE Education Society, a DuPont Young Faculty Award, a National Science Foundation Presidential Young Investigator Award, Newport Research Award, and three teaching awards, and she was a Hewlett-Packard Fellow. She has organized and served on numerous conference committees and served on the Board of Directors of the Optical Society of America as the Chair of the Engineering Council and on the IEEE Lasers and Electro-Optics Society (LEOS) Board of Governors as an elected member, and as the Vice President of Conferences for IEEE LEOS. She has also served as the elected Chair, Vice Chair, Secretary, and Treasurer of the Atlanta IEEE Section. 